PROCEEDINGS OF THE

AMERICAN MATHEMATICAL SOCIETY

Volume 131, Number 9, Pages 2903-2909

S 0002-9939(03)07132-6

Article electronically published on April 9, 2003

\title{
ALMOST HERMITIAN STRUCTURES INDUCED FROM A KÄHLER STRUCTURE WHICH HAS CONSTANT HOLOMORPHIC SECTIONAL CURVATURE
}

\author{
TAKUJI SATO
}

(Communicated by Mohan Ramachandran)

\begin{abstract}
We obtain a non-Kähler almost Hermitian manifold of constant holomorphic sectional curvature by changing the almost complex structure in a Kähler manifold of constant holomorphic sectional curvature.
\end{abstract}

\section{INTRODUCTION}

Let $M=(M, J, g)$ be an almost Hermitian manifold. The holomorphic sectional curvature $H=H(x)$ of $M$ can be regarded as a differentiable function on the unit tangent bundle $U(M)$ of $M$. If the function $H$ is constant along each fiber, then $M$ is called a space of pointwise constant holomorphic sectional curvature. In particular, if $H$ is constant on the whole of $U(M)$, then $M$ is called a space of constant holomorphic sectional curvature.

It is well known that the complex projective space $\mathbb{C} P^{n}$ and the open unit ball $D^{2 n}$ in $\mathbb{C}^{n}$ are equipped with the Kähler structure of constant holomorphic sectional curvature (e.g. [4). The 6-dimensional sphere $S^{6}$ is known as the nearly Kähler manifold of constant holomorphic sectional curvature (in fact, of constant sectional curvature). For the examples of almost Hermitian manifolds with pointwise constant holomorphic sectional curvature, we refer to A. Gray and L. Vanhecke [2], P. Nurowski and M. Przanowski [3] and T. Sato [6], [7, 8].

However, it seems to the author that the non-Kähler examples of almost Hermitian manifolds which have constant holomorphic sectional curvature are not known, except for the one of constant sectional curvature. The purpose of the present paper is to provide such examples. Namely, we show that there exist non-Kähler almost Hermitian manifolds of constant holomorphic sectional curvature which are not of constant curvature. Our examples are obtained on a coordinate neighborhood of $\mathbb{C} P^{n}$ or on $D^{2 n}$ by changing the almost complex structure. In a similar way, we can also obtain Einstein and $*$-Einstein Hermitian surfaces.

\section{Preliminaries}

Let $M=(M, J, g)$ be an $m(=2 n)$-dimensional almost Hermitian manifold with an almost Hermitian structure $(J, g)$. We denote by $N$ the Nijenhuis tensor of $M$

Received by the editors March 15, 2001.

2000 Mathematics Subject Classification. Primary 53B35, 53C15, 53C55.

Key words and phrases. Almost Hermitian manifold, holomorphic sectional curvature.

(C)2003 American Mathematical Society 
defined by $N(X, Y)=[J X, J Y]-[X, Y]-J[J X, Y]-J[X, J Y]$ for $X, Y \in \mathfrak{X}(M)$, where $\mathfrak{X}(M)$ is the Lie algebra of all smooth vector fields on $M$. The Nijenhuis tensor $N$ satisfies

$$
N(J X, Y)=N(X, J Y)=-J N(X, Y), \quad X, Y \in \mathfrak{X}(M) .
$$

Further we denote by $\nabla, R, \rho, \tau, \rho^{*}$ and $\tau^{*}$ the Riemannian connection, the Riemannian curvature tensor, the Ricci tensor, the scalar curvature, the Ricci $*$-tensor and the $*$-scalar curvature of $M$, respectively. The Ricci $*$-tensor $\rho^{*}$ and the $*$-scalar curvature are defined by

$$
\begin{aligned}
\rho^{*}(x, y) & =\text { trace of }[z \mapsto R(x, J z) J y], \\
\tau^{*} & =\text { trace of } \rho^{*},
\end{aligned}
$$

where $x, y, z \in T_{p} M, p \in M$.

An almost Hermitian manifold $M$ is called a $*$-Einstein manifold if it satisfies $\rho^{*}=\lambda^{*} g$ for some constant $\lambda^{*}$ on $M$.

Now, we define the tensor field $G$ by

$$
G(X, Y, Z, W)=R(X, Y, Z, W)-R(X, Y, J Z, J W) .
$$

The tensor field $G$ plays an important role in the study of almost Hermitian manifolds (cf. A. Gray [1, F. Tricerri-L. Vanhecke 9], T. Sato [5], etc.). For the Kähler manifolds, it is obvious that $G=0$. It is easy to see that the condition

$$
G(X, Y, Z, W)=G(Z, W, X, Y), \quad \text { for } X, Y, Z, W \in \mathfrak{X}(M),
$$

is equivalent to the curvature $J$-invariant condition:

$$
R(J X, J Y, J Z, J W)=R(X, Y, Z, W),
$$

for $X, Y, Z, W \in \mathfrak{X}(M)$. An almost Hermitian manifold $M$ satisfying (2.3) is called an $R K$-manifold.

By using the tensor $G$, we have obtained the following characterization of almost Hermitian manifolds of pointwise constant holomorphic sectional curvature:

Proposition 2.1 ([5]). Let $M$ be an RK-manifold of pointwise constant holomorphic sectional curvature $c=c(p)(p \in M)$. Then

$$
R(x, y, z, w)=\frac{c(p)}{4} R_{0}(x, y, z, w)+P(x, y, z, w),
$$

where

$$
\begin{aligned}
& R_{0}(x, y, z, w)=g(x, w) g(y, z)-g(x, z) g(y, w) \\
& \quad+g(x, J w) g(y, J z)-g(x, J z) g(y, J w)-2 g(x, J y) g(z, J w), \\
& \begin{aligned}
P(x, y, z, w) \\
=\frac{1}{24}\{10 G(x, y, z, w)+5 G(x, z, y, w)-5 G(x, w, y, z) \\
\quad+2 G(x, J y, z, J w)+G(x, J z, y, J w)-G(x, J w, y, J z)\} .
\end{aligned}
\end{aligned}
$$

In an $R K$-manifold of constant holomorphic sectional curvature, from (2.4) we have

$$
\begin{aligned}
& \rho(x, y)+3 \rho^{*}(x, y)=(m+2) c(p) g(x, y), \\
& \tau+3 \tau^{*}=m(m+2) c(p) .
\end{aligned}
$$




\section{Examples of almost HeRmitian manifold WITH CONSTANT HOLOMORPHIC SECTIONAL CURVATURE}

We first show the following

Theorem 3.1. Let $\left(M, J_{0}, g\right)$ be a Kähler manifold of constant holomorphic sectional curvature c. If $(J, g)$ is an almost Hermitian structure on $M$ satisfying $J J_{0}=-J_{0} J$, then $(M, J, g)$ is an $R K$-manifold of constant holomorphic sectional curvature $c / 4$ and $\rho^{*}=0$.

Proof. It is well known that the curvature tensor of $\left(M, J_{0}, g\right)$ is given by

$$
\begin{gathered}
R(x, y, z, w)=\frac{c}{4}\left\{g(x, w) g(y, z)-g(x, z) g(y, w)+g\left(x, J_{0} w\right) g\left(y, J_{0} z\right)\right. \\
\left.-g\left(x, J_{0} z\right) g\left(y, J_{0} w\right)-2 g\left(x, J_{0} y\right) g\left(z, J_{0} w\right)\right\}
\end{gathered}
$$

for any $x, y, z, w \in T_{p} M$ (4]). Since $(J, g)$ is almost Hermitian and $J$ is anticommuting with $J_{0}$, it is easy to see that $R(J x, J y, J z, J w)=R(x, y, z, w)$, hence $(M, J, g)$ is an $R K$-manifold.

By (3.1), we have

$$
\begin{aligned}
& R(x, y, J z, J w) \\
& =\frac{c}{4}\left\{\begin{array}{l}
g(x, J w) g(y, J z)-g(x, J z) g(y, J w)+g\left(x, J_{0} J w\right) g\left(y, J_{0} J z\right) \\
\left.\quad-g\left(x, J_{0} J z\right) g\left(y, J_{0} J w\right)+2 g\left(x, J_{0} y\right) g\left(z, J_{0} w\right)\right\}
\end{array}\right.
\end{aligned}
$$

and

$$
\begin{aligned}
& G(x, y, z, w)=R(x, y, z, w)-R(x, y, J z, J w) \\
& \quad=\frac{c}{4}\left\{g(x, w) g(y, z)-g(x, z) g(y, w)+g\left(x, J_{0} w\right) g\left(y, J_{0} z\right)\right. \\
& \quad-g\left(x, J_{0} z\right) g\left(y, J_{0} w\right)-g(x, J w) g(y, J z)+g(x, J z) g(y, J w) \\
& \left.\quad-g\left(x, J_{0} J w\right) g\left(y, J_{0} J z\right)+g\left(x, J_{0} J z\right) g\left(y, J_{0} J w\right)-4 g\left(x, J_{0} y\right) g\left(z, J_{0} w\right)\right\} .
\end{aligned}
$$

From (3.3), we obtain

$$
\begin{aligned}
10 G & (x, y, z, w)+5 G(x, z, y, w)-5 G(x, w, y, z) \\
& +2 G(x, J y, z, J w)+G(x, J z, y, J w)-G(x, J w, y, J z) \\
& =\frac{3 c}{2}\left\{3 g(x, w) g(y, z)-3 g(x, z) g(y, w)+4 g\left(x, J_{0} w\right) g\left(y, J_{0} z\right)\right. \\
& -4 g\left(x, J_{0} z\right) g\left(y, J_{0} w\right)-8 g\left(x, J_{0} y\right) g\left(z, J_{0} w\right) \\
& -g(x, J w) g(y, J z)+g(x, J z) g(y, J w)+2 g(x, J y) g(z, J w)\} .
\end{aligned}
$$

This can be written as

$$
\begin{aligned}
& P(x, y, z, w)=R(x, y, z, w) \\
& \quad-\frac{c}{16}\{g(x, w) g(y, z)-g(x, z) g(y, w)+g(x, J w) g(y, J z) \\
& \quad-g(x, J z) g(y, J w)-2 g(x, J y) g(z, J w)\} .
\end{aligned}
$$

Therefore $(M, J, g)$ is an $R K$-manifold of constant holomorphic sectional curvature $c / 4$ by virtue of Proposition 2.1 . 
Next, we shall show that the Ricci $*$-tensor $\rho^{*}$ of $(M, J, g)$ vanishes. Let $\left\{e_{i}\right\}$ be an orthonormal basis of $T_{p} M$. By definition and (3.1), we have

$$
\begin{aligned}
\rho^{*}(y, z) & =\sum_{i=1}^{m} R\left(e_{i}, y, J z, J e_{i}\right) \\
& =\frac{c}{4} \sum_{i=1}^{m}\left\{g\left(e_{i}, J e_{i}\right) g(y, J z)-g\left(e_{i}, J z\right) g\left(y, J e_{i}\right)\right. \\
& +g\left(e_{i}, J_{0} J e_{i}\right) g\left(y, J_{0} J z\right)-g\left(e_{i}, J_{0} J z\right) g\left(y, J_{0} J e_{i}\right) \\
& \left.-2 g\left(e_{i}, J_{0} y\right) g\left(J z, J_{0} J e_{i}\right)\right\} \\
& =\frac{c}{4} \sum_{i=1}^{m}\left\{g\left(e_{i}, J z\right) g\left(J y, e_{i}\right)+g\left(e_{i}, J_{0} J z\right) g\left(J_{0} J y, e_{i}\right)\right. \\
& \left.+2 g\left(e_{i}, J_{0} y\right) g\left(z, J_{0} e_{i}\right)\right\} \\
& =\frac{c}{4}\left\{g(J y, J z)+g\left(J_{0} J y, J_{0} J z\right)-2 g\left(J_{0} y, J_{0} z\right)\right\}=0 .
\end{aligned}
$$

Now, we shall provide an example of an almost Hermitian manifold which has constant holomorphic sectional curvature. Let $M=\mathbb{R}^{2 n}$ with the coordinate system $\left(x_{1}, x_{2}, \cdots, x_{2 n}\right)$. We define a natural complex structure $J_{0}$ by

$$
J_{0}\left(\frac{\partial}{\partial x_{2 i-1}}\right)=\frac{\partial}{\partial x_{2 i}}, \quad J_{0}\left(\frac{\partial}{\partial x_{2 i}}\right)=-\frac{\partial}{\partial x_{2 i-1}},
$$

and a Riemannian metric $g=\left(g_{i j}\right)$ by

$$
g_{i j}=\frac{4}{c A^{2}}\left(A \delta_{i j}-x_{i} x_{j}-x_{\bar{i}} x_{\bar{j}}\right),
$$

where $c>0, A=1+\sum_{i=1}^{2 n} x_{i}^{2}$ and we denote $x_{\overline{2 i-1}}=x_{2 i}, x_{\overline{2 i}}=-x_{2 i-1}$. The metric of (3.8) is nothing but the real representation of the Fubini-Study metric on a coordinate neighborhood of $\mathbb{C} P^{n}$. Therefore $\left(\mathbb{R}^{2 n}, J_{0}, g\right)$ is a Kähler manifold of constant holomorphic sectional curvature $c$ (cf. [4]).

Let $\left\{e_{1}, e_{2}=J_{0} e_{1}, \cdots, e_{2 n-1}, e_{2 n}=J_{0} e_{2 n-1}\right\}$ be a unitary frame on $M$ with respect to $\left(J_{0}, g\right)$. If we adopt the notation $e_{\overline{2 i-1}}=e_{2 i}, e_{\overline{2 i}}=-e_{2 i-1}$, then it can be written as $J_{0} e_{i}=e_{\bar{i}}$. We define a new almost complex structure $J$ by

$$
J e_{i}=\sum_{k=1}^{2 n} J_{i}{ }^{k} e_{k},
$$

where the coefficients $J_{i}{ }^{k}$ satisfy

$$
\begin{aligned}
\sum_{k=1}^{2 n}{J_{i}}^{k}{J_{k}}^{j} & =-\delta_{i}^{j}, \\
\sum_{k=1}^{2 n}{J_{i}}^{k} J_{j}{ }^{k} & =\delta_{i j}, \\
J_{i}{ }^{\bar{k}} & ={J_{\bar{i}}}^{k} .
\end{aligned}
$$


From (3.10) and (3.11), $(J, g)$ is almost Hermitian and (3.12) implies that $J$ anticommutes with $J_{0}$. Indeed,

$$
\begin{aligned}
J_{0} J e_{i} & =J_{0}\left(\sum_{k=1}^{2 n} J_{i}{ }^{k} e_{k}\right)=\sum_{k=1}^{2 n} J_{i}{ }^{k} J_{0} e_{k}=\sum_{k=1}^{2 n} J_{i}{ }^{k} e_{\bar{k}} \\
& =-\sum_{k=1}^{2 n} J_{i}{ }^{\bar{k}} e_{k}=-\sum_{k=1}^{2 n} J_{\bar{i}}{ }^{k} e_{k}=-J e_{\bar{i}} \\
& =-J J_{0} e_{i} .
\end{aligned}
$$

For example, the matrices $\left(J_{i}{ }^{k}\right)$ are given by the following form: when $n=2$,

$$
\left(J_{i}{ }^{k}\right)=\left(\begin{array}{cc}
0 & -A_{0} \\
A_{0} & 0
\end{array}\right)
$$

where $A_{0}=\left(\begin{array}{cc}a & b \\ b & -a\end{array}\right)$ and $a^{2}+b^{2}=1$, and when $n=3$,

$$
\left(J_{i}{ }^{k}\right)=\left(\begin{array}{ccc}
0 & -A_{1} & -A_{2} \\
A_{1} & 0 & -A_{3} \\
A_{2} & A_{3} & 0
\end{array}\right),
$$

where $A_{i}=\left(\begin{array}{cc}a_{i} & b_{i} \\ b_{i} & -a_{i}\end{array}\right)$ and $a_{i}^{2}+b_{i}^{2}=\frac{1}{2}(i=1,2,3)$.

Thus we can conclude that $(M, J, g)$ is an almost Hermitian manifold of constant holomorphic sectional curvature $c / 4(>0)$ by virtue of Theorem 3.1

Moreover, we have

$$
\begin{array}{ll}
\rho=\frac{n+1}{2} c g, & \tau=n(n+1) c\left(=m(m+2) \frac{c}{4}\right), \\
\rho^{*}=0, & \tau^{*}=0 .
\end{array}
$$

Since $\tau \neq \tau^{*}$, we see that $(M, J, g)$ is non-Kähler.

Next, let $D^{2 n}=\left\{\left(x_{1}, x_{2}, \cdots, x_{2 n-1}, x_{2 n}\right) \in \mathbb{R}^{2 n} \mid \sum_{i=1}^{2 n} x_{i}^{2}<1\right\}$ be the unit ball in $\mathbb{R}^{2 n}$, and let $J_{0}$ be the natural complex structure. We define a Riemannian metric $g=\left(g_{i j}\right)$ on $D^{2 n}$ by

$$
g_{i j}=-\frac{4}{c A^{2}}\left(A \delta_{i j}+x_{i} x_{j}+x_{i} x_{\bar{j}}\right),
$$

where $c<0$ and $A=1-\sum_{i=1}^{2 n} x_{i}^{2}$. Then it is well known that $\left(D^{2 n}, J_{0}, g\right)$ is a Kähler manifold of constant holomorphic sectional curvature $c$ (cf. [4]). In the same way as above, if we define a new almost complex structure $J$ by (3.9) $\sim(3.12$, then $\left(D^{2 n}, J, g\right)$ is a non-Kähler $R K$-manifold of constant holomorphic sectional curvature $c / 4(<0)$ and $\rho^{*}$ vanishes. Consequently, we have the following

Theorem 3.2. (1) For any positive number $c, \mathbb{R}^{2 n}$ admits a non-Kähler almost Hermitian structure $(J, g)$ of constant holomorphic sectional curvature $c / 4$, which is Einstein and Ricci *-flat, but not constant curvature.

(2) For any negative number $c$, the open unit ball $D^{2 n}$ in $\mathbb{R}^{2 n}$ admits a nonKähler almost Hermitian structure $(J, g)$ of constant holomorphic sectional curvature $c / 4$, which is Einstein and Ricci $*$-flat, but not constant curvature. 


\section{Examples of Einstein AND *-Einstein Hermitian surfaces}

In this section, we shall give an example of an Einstein and $*$-Einstein Hermitian surface which is not Kählerian.

Let $\left(\mathbb{R}^{4}, J_{0}, g\right),\left(D^{4}, J_{0}, g\right)$ be the Kähler surfaces of constant holomorphic sectional curvature in $\S 3$. We denote by $M^{4}$ the manifold $\mathbb{R}^{4}$ or $D^{4}$. We obtain a unitary frame $\left\{e_{i}\right\}$ on $\left(M^{4}, J_{0}, g\right)$ as follows:

$$
\begin{aligned}
& e_{1}=\frac{\sqrt{ \pm c} A}{2 \sqrt{B}} \frac{\partial}{\partial x_{1}}, \\
& e_{2}=\frac{\sqrt{ \pm c} A}{2 \sqrt{B}} \frac{\partial}{\partial x_{2}}, \\
& e_{3}=\frac{\sqrt{ \pm c A}}{2 \sqrt{B}}\left\{ \pm\left(x_{1} x_{3}+x_{\overline{1}} x_{\overline{3}}\right) \frac{\partial}{\partial x_{1}} \pm\left(x_{2} x_{3}+x_{\overline{2}} x_{\overline{3}}\right) \frac{\partial}{\partial x_{2}}+B \frac{\partial}{\partial x_{3}}\right\}, \\
& e_{4}=\frac{\sqrt{ \pm c A}}{2 \sqrt{B}}\left\{ \pm\left(x_{1} x_{4}+x_{\overline{1}} x_{\overline{4}}\right) \frac{\partial}{\partial x_{1}} \pm\left(x_{2} x_{4}+x_{\overline{2}} x_{\overline{4}}\right) \frac{\partial}{\partial x_{2}}+B \frac{\partial}{\partial x_{4}}\right\},
\end{aligned}
$$

where $A=1 \pm \sum_{i=1}^{4} x_{i}^{2}, B=1 \pm \sum_{i=3}^{4} x_{i}^{2}$ and the \pm sign corresponds respectively to the $\mathbb{R}^{4}$ and $D^{4}$ cases.

By straightforward computations, we have

$$
\begin{aligned}
& {\left[e_{1}, e_{2}\right]= \pm \frac{\sqrt{ \pm c}}{\sqrt{B}}\left(-x_{2} e_{1}+x_{1} e_{2}\right),} \\
& {\left[e_{1}, e_{3}\right]= \pm \frac{\sqrt{ \pm c}}{2 \sqrt{B}}\left(-x_{4} \sqrt{A} e_{2}+x_{1} e_{3}\right),} \\
& {\left[e_{1}, e_{4}\right]= \pm \frac{\sqrt{ \pm c}}{2 \sqrt{B}}\left(x_{3} \sqrt{A} e_{2}+x_{1} e_{4}\right),} \\
& {\left[e_{2}, e_{3}\right]= \pm \frac{\sqrt{ \pm c}}{2 \sqrt{B}}\left(x_{4} \sqrt{A} e_{1}+x_{2} e_{3}\right),} \\
& {\left[e_{2}, e_{4}\right]= \pm \frac{\sqrt{ \pm c}}{2 \sqrt{B}}\left(-x_{3} \sqrt{A} e_{1}+x_{2} e_{4}\right),} \\
& {\left[e_{3}, e_{4}\right]= \pm \frac{\sqrt{ \pm c}}{\sqrt{B}}\left(-x_{2} e_{1}+x_{1} e_{2}-x_{4} \sqrt{A} e_{3}+x_{3} \sqrt{A} e_{4}\right) .}
\end{aligned}
$$

Now, we define a new almost complex structure $J$ on $M^{4}$ by

$$
J e_{1}=e_{2}, J e_{2}=-e_{1}, J e_{3}=-e_{4}, J e_{4}=e_{3} .
$$

Then it is obvious that $(J, g)$ is almost Hermitian. Further we see that the Nijenhuis tensor $N_{J}$ of $J$ vanishes. Indeed, by (4.2) and (4.3)

$$
\begin{aligned}
N_{J}\left(e_{1}, e_{3}\right) & =\left[J e_{1}, J e_{3}\right]-\left[e_{1}, e_{3}\right]-J\left[J e_{1}, e_{3}\right]-J\left[e_{1}, J e_{3}\right] \\
& =-\left[e_{2}, e_{4}\right]-\left[e_{1}, e_{3}\right]-J\left[e_{2}, e_{3}\right]+J\left[e_{1}, e_{4}\right]=0 .
\end{aligned}
$$

It follows that $N_{J}\left(e_{i}, e_{j}\right)=0$ for all $i, j$ from (2.1). 
The curvature tensor of $\left(M^{4}, J, g\right)$ is given by (3.1) and $g$ is an Einstein metric. By considering that $J_{0} J=J J_{0}$ and $\sum_{i=1}^{4} g\left(e_{i}, J_{0} J e_{i}\right)=0$, we have

$$
\begin{aligned}
\rho^{*}(y, z) & =\sum_{i=1}^{4} R\left(e_{i}, y, J z, J e_{i}\right) \\
& =\frac{c}{4} \sum_{i=1}^{4}\left\{g\left(e_{i}, J e_{i}\right) g(y, J z)-g\left(e_{i}, J z\right) g\left(y, J e_{i}\right)\right. \\
& +g\left(e_{i}, J_{0} J e_{i}\right) g\left(y, J_{0} J z\right)-g\left(e_{i}, J_{0} J z\right) g\left(y, J_{0} J e_{i}\right) \\
& \left.-2 g\left(e_{i}, J_{0} y\right) g\left(J z, J_{0} J e_{i}\right)\right\} \\
& =\frac{c}{4} \sum_{i=1}^{4}\left\{g\left(e_{i}, J z\right) g\left(J y, e_{i}\right)-g\left(e_{i}, J_{0} J z\right) g\left(J_{0} J y, e_{i}\right)\right. \\
& \left.-2 g\left(e_{i}, J_{0} y\right) g\left(z, J_{0} e_{i}\right)\right\} \\
& =\frac{c}{4}\left\{g(J y, J z)-g\left(J_{0} J y, J_{0} J z\right)+2 g\left(J_{0} y, J_{0} z\right)\right\} \\
& =\frac{c}{2} g(y, z) .
\end{aligned}
$$

Since

$$
\begin{array}{ll}
\rho=\frac{3}{2} c g, & \tau=6 c, \\
\rho^{*}=\frac{c}{2} g, & \tau^{*}=2 c,
\end{array}
$$

$\left(M^{4}, J, g\right)$ is non-Kähler, Einstein and $*$-Einstein.

Summing up the above arguments, we obtain the following

Theorem 4.1. The Euclidean 4-space $\mathbb{R}^{4}$ and the open unit ball $D^{4}$ admit a non-Kähler Hermitian structure $(J, g)$, which is Einstein and $*$-Einstein.

\section{REFERENCES}

[1] A. Gray, Curvature identities for Hermitian and almost Hermitian manifolds, Tôhoku Math. J., 28(1976), 601-612. MR 55:9005

[2] A. Gray and L. Vanhecke, Almost Hermitian manifolds with constant holomorphic sectional curvature, Časopis pro pěstování mat., 104(1979), 170-179. MR 80f:53039

[3] P. Nurowski and M. Przanowski, A four-dimensional example of a Ricci-flat metric admitting almost-Kähler non-Kähler structure, Classical Quantum Gravity, 16(1999), L9-L13. MR 2000a:53073

[4] S. Kobayashi and K. Nomizu, Foundations of differential geometry, I, II, Interscience Publishers, New York, 1963, 1969. MR 27:2945] MR 38:6501

[5] T. Sato, On some almost Hermitian manifolds with constant holomorphic sectional curvature, Kyungpook Math. J., 29(1989), 11-25. MR 91d:53052

[6] T. Sato, Some remarks on almost Kähler 4-manifolds of pointwise constant holomorphic sectional curvature, Kodai Math. J., 22(1999), 286-303. MR 2001c:53037

[7] T. Sato, An example of an almost Kähler manifold with pointwise constant holomorphic sectional curvature, Tokyo J. Math., 23(2000), 387-401. MR 2002b:53067

[8] T. Sato, Some examples of almost Kähler 4-manifolds, Balkan J. Geom. Appl., 5(2000), 113-137. MR 2003c:53059

[9] F. Tricerri and L. Vanhecke, Curvature tensors on almost Hermitian manifolds, Trans. Amer. Math. Soc., 267(1981), 365-398. MR 82j:53071

Faculty of Engineering, Kanazawa University, Kanazawa, Japan

E-mail address: tsato@t.kanazawa-u.ac.jp 